PROCEEDINGS OF THE

AMERICAN MATHEMATICAL SOCIETY

Volume 130, Number 9, Pages 2559-2567

S 0002-9939(02)06396-7

Article electronically published on March 12, 2002

\title{
ONE SIDED IDEALS AND CARLSON'S THEOREM
}

\author{
NEIL HINDMAN AND RANDALL MCCUTCHEON
}

(Communicated by John R. Stembridge)

\begin{abstract}
Using left ideals, right ideals, and the smallest two sided ideal in a compact right topological semigroup, we derive an extension of the Main Lemma to Carlson's Theorem. This extension involves an infinite sequence of variable words over a finite alphabet, some of which are required to have the variable as the first letter and others of which are required to have the variable as the last letter.
\end{abstract}

\section{INTRODUCTION}

In 1988 T. Carlson published a Ramsey Theoretic result 2, Theorem 2] which has as corollaries many earlier results in Ramsey Theory. (See [8, Section 18.4] for a relatively short presentation of Carlson's Theorem and some of its consequences.) Experience suggests that Carlson's "Main Lemma" 2, Lemma 5.9] implies those Ramsey Theoretic corollaries of his theorem in which a finite collection of finite objects is partitioned into finitely many classes. We shall state this Main Lemma after introducing some necessary terminology.

For $k \in \mathbb{N}$, the set of positive integers, let $\mathcal{W}_{k}$ be the free semigroup with identity $e$ on the alphabet $\{1,2, \ldots, k\}$. That is, $\mathcal{W}_{k}$ consists of all "words with letters from $\{1,2, \ldots, k\}$ " (i.e. functions whose domain is an initial segment of $\mathbb{N}$ and whose range is contained in $\{1,2, \ldots, k\})$ together with the empty word, with the operation of concatenation. A variable word over $\mathcal{W}_{k}$ is a word on the alphabet $\{1,2, \ldots, k\} \cup\{v\}$ in which $v$ occurs, where $v$ is a "variable" not in $\{1,2, \ldots$, $k\}$. Given a variable word $w$ over $\mathcal{W}_{k}$, and $t \in\{1,2, \ldots, k\}, w(t)$ has its obvious meaning, namely the result of replacing all occurrences of $v$ with $t$. (There is a potential conflict here with the formal viewpoint which takes $w$ to be a function. If we have occasion to need the value of the function $w$ at $t$ we will denote it as $w_{t}$.)

1.1. Definition. Let $k \in \mathbb{N}$ and let $\left\langle w_{n}\right\rangle_{n=1}^{\infty}$ be a sequence of variable words over $\mathcal{W}_{k}$. The sequence $\left\langle t_{n}\right\rangle_{n=1}^{\infty}$ is a variable reduction of $\left\langle w_{n}\right\rangle_{n=1}^{\infty}$ if and only if there exist an increasing function $g: \mathbb{N} \rightarrow \mathbb{N}$ and a function $f: \mathbb{N} \rightarrow\{1,2, \ldots, k\} \cup\{v\}$ such that:

(1) $g(1)=1$,

Received by the editors October 16, 2000 and, in revised form, April 19, 2001.

1991 Mathematics Subject Classification. Primary 05D10; Secondary 22A15, 22A30, 54D30.

The first author acknowledges support received from the National Science Foundation (USA) via grant DMS-0070593.

The second author acknowledges support received from the National Science Foundation via a post doctoral fellowship administered by the University of Maryland.

(C)2002 American Mathematical Society 
(2) for each $n \in \mathbb{N}, v \in f[\{g(n), g(n)+1, \ldots, g(n+1)-1\}]$, and

(3) for each $n \in \mathbb{N}, t_{n}=\prod_{i=g(n)}^{g(n+1)-1} w_{i}(f(i))$, where the product is in increasing order of indices.

1.2. Theorem (Carlson). Let $k \in \mathbb{N}$, let the set of variable words over $\mathcal{W}_{k}$ be partitioned into finitely many classes, and let $\left\langle w_{n}\right\rangle_{n=1}^{\infty}$ be a sequence of variable words. Then there exists a variable reduction $\left\langle t_{n}\right\rangle_{n=1}^{\infty}$ of $\left\langle w_{n}\right\rangle_{n=1}^{\infty}$ such that all expressions of the form $\prod_{i=1}^{n} t_{i}(f(i))$, where $n \in \mathbb{N}, f:\{1,2, \ldots, n\} \rightarrow\{1,2, \ldots$, $k\} \cup\{v\}$, and $v \in$ range $(f)$, lie in the same cell of the partition.

Proof. [2] Lemma 5.9].

Somewhat earlier, T. Carlson and S. Simpson had established a similar result which partitioned $\mathcal{W}_{k}$ rather than the variable words over $\mathcal{W}_{k}$ and required that most of the variable words used must have $v$ as the leftmost letter. Such words are left variable words. Similarly, a right variable word must have the variable $v$ as its rightmost letter.

1.3. Theorem (Carlson-Simpson). Let $k \in \mathbb{N}$ and let $\mathcal{W}_{k}$ be partitioned into finitely many classes. Then there exists a sequence $\left\langle w_{n}\right\rangle_{n=1}^{\infty}$ of variable words over $\mathcal{W}_{k}$ such that for every $n>1, w_{n}$ is a left variable word, and all expressions of the form $\prod_{i=1}^{n} w_{i}(f(i))$, where $n \in \mathbb{N}$ and $f:\{1,2, \ldots, n\} \rightarrow\{1,2, \ldots, k\}$, lie in the same cell of the partition.

Proof. [3] Theorem 6.3].

In [1, V. Bergelson, A. Blass, and the first author of the current paper established a generalization of Theorem 1.2 by utilizing the algebraic structure of the StoneCech compactification of a discrete semigroup. They could not, however, extend by these methods Theorem 1.3 The reasons involve the algebraic constructs used in the proof, which we pause now to introduce.

Given a discrete semigroup $(S, \cdot)$, we take the points of $\beta S$ to be the ultrafilters on $S$, the principal ultrafilters being identified with the points of $S$. Given a set $A \subseteq S, \bar{A}=\{p \in \beta S: A \in p\}$. The set $\{\bar{A}: A \subseteq S\}$ is a basis for the open sets (as well as a basis for the closed sets) of $\beta S$.

There is a natural extension of the operation - of $S$ to $\beta S$ making $\beta S$ a compact right topological semigroup with $S$ contained in its topological center. This says that for each $p \in \beta S$ the function $\rho_{p}: \beta S \rightarrow \beta S$ is continuous and for each $x \in S$, the function $\lambda_{x}: \beta S \rightarrow \beta S$ is continuous, where $\rho_{p}(q)=q \cdot p$ and $\lambda_{x}(q)=x \cdot q$. The operation is characterized by the fact that for any $p$ and $q$ in $\beta S$ and any $A \subseteq S$, $A \in p \cdot q$ if and only if $\left\{x \in S: x^{-1} A \in q\right\} \in p$, where $x^{-1} A=\{y \in S: x \cdot y \in A\}$. See 8 for an elementary introduction to the semigroup $\beta S$.

Any compact Hausdorff right topological semigroup $(T, \cdot)$ has a smallest two sided ideal $K(T)$ which is the union of all of the minimal left ideals of $T$, each of which is closed, and is also the union of all of the minimal right ideals of $T$ [8, Theorem 2.8]. Given any minimal left ideal $L$ and any minimal right ideal $R$, $L \cap R$ is a group (and in particular has an idempotent) [8, Theorem 2.7]. There is a partial ordering of the idempotents of $T$ determined by $p \leq q$ if and only if $p=p \cdot q=q \cdot p$. An idempotent $p$ is minimal with respect to this order if and only if $p \in K(T)$ [ 8 , Theorem 1.59]. Such an idempotent is called simply "minimal".

Members of minimal idempotents in $\beta S$ are the central subsets of $S$. Central sets were introduced by H. Furstenberg in [4] and defined in terms of notions of 
topological dynamics. These sets enjoy very strong combinatorial properties. (See [4, Proposition 8.21] or [8, Chapter 14].) See [8, Theorem 19.27] for a proof of the equivalence of the definition of "central" in terms of $\beta S$ with the original dynamical definition.

The basic algebraic fact used in [1] (as well as several other papers) is that any two sided ideal in a compact right topological semigroup $T$ contains $K(T)$. While variable words over $\mathcal{W}_{k}$ yield a two sided ideal in an appropriately chosen compact right topological semigroup, left variable words and right variable words do not.

Left variable words do, however, correspond naturally to a right ideal (and right variable words correspond to a left ideal). In [7] we were able to use these natural left and right ideals to obtain a generalization of Theorem 1.3 involving both left and right variable words. In the current paper we use similar left and right ideals to obtain a generalization of Theorem 1.2 which involves both left and right variable words.

\section{Extending Carlson's Main Lemma}

We shall have throughout this section a fixed $k \in \mathbb{N}$. We begin with the following simple lemma from semigroup theory.

2.1. Lemma. Let $S$ be a semigroup which has a minimal left ideal which contains an idempotent. Let $L$ be a left ideal of $S$, let $R$ be a right ideal of $S$, and let e be any idempotent in $S$. There is a minimal idempotent $m \in L e \cap e R$. Necessarily $m \leq e$.

Proof. Notice that if $m \in L e$, then $m e=m$ and if $m \in e R$, then $e m=m$. Thus any idempotent $m \in L e \cap e R$ satisfies $m \leq e$.

By [8, Lemma 1.57] $S$ has a minimal right ideal which contains an idempotent. Thus, by [8] Corollary 1.47] we may presume that $L$ is a minimal left ideal and $R$ is a minimal right ideal. By [8, Theorem 1.46] $L e$ is a minimal left ideal and $e R$ is a minimal right ideal and so, by [8, Theorem 1.61], Le $\cap e R$ is a group. Let $m$ be the identity of $L e \cap e R$.

Given a set $X$, we denote the set of finite nonempty subsets of $X$ by $\mathcal{P}_{f}(X)$.

2.2. Lemma. Let $S$ be a discrete semigroup, let $A \subseteq \chi_{j=1}^{k+1} S$, and let $Z=$ $\chi_{j=1}^{k+1} \beta S$. Assume that $\left(y_{1}, y_{2}, \ldots, y_{k+1}\right) \in c \ell_{Z}(A), C \subseteq S, W \in \mathcal{P}_{f}(S)$, and $L \in \mathcal{P}_{f}(\beta S)$. Then there exists $\left(a_{1}, a_{2}, \ldots, a_{k+1}\right) \in A$ such that, for all $l \in L$, all $u \in W$, and all $i \in\{1,2, \ldots, k+1\}$,

$$
\left(u a_{i}\right)^{-1} C \in l \Leftrightarrow u^{-1} C \in y_{i} l .
$$

Proof. For $l \in L, u \in W$, and $i \in\{1,2, \ldots, k+1\}$, let

$$
C_{i, u, l}= \begin{cases}C & \text { if } C \in u y_{i} l, \\ S \backslash C & \text { if } C \notin u y_{i} l .\end{cases}
$$

Then $\overline{C_{i, u, l}}$ is a neighborhood of $u y_{i} l=\lambda_{u}\left(\rho_{l}\left(y_{i}\right)\right)$, so pick a member $U_{i, u, l}$ of $y_{i}$ such that $\lambda_{u}\left[\rho_{l}\left[\overline{U_{i, u, l}}\right]\right] \subseteq \overline{C_{i, u, l}}$.

For $i \in\{1,2, \ldots, k+1\}$, let $N_{i}=\bigcap_{l \in L} \bigcap_{u \in W} U_{i, u, l}$. Pick $\left(a_{1}, a_{2}, \ldots, a_{k+1}\right) \in$ $A \cap \chi_{i=1}^{k+1} N_{i}$. 
2.3. Definition. (a) $Y=\left(X_{j=1}^{k} \beta \mathcal{W}_{k}\right) \times \beta \mathcal{W}_{k+1}$.

(b) $Z=X_{j=1}^{k+1} \beta \mathcal{W}_{k+1}$.

(c) $I=\left\{(w(1), w(2), \ldots, w(k+1)): w\right.$ is a variable word over $\left.\mathcal{W}_{k}\right\}$.

(d) $J=\left\{(w(1), w(2), \ldots, w(k+1)): w\right.$ is a left variable word over $\left.\mathcal{W}_{k}\right\}$.

(e) $H=\left\{(w(1), w(2), \ldots, w(k+1)): w\right.$ is a right variable word over $\left.\mathcal{W}_{k}\right\}$.

(f) $E=I \cup\left\{(w, w, \ldots, w): w \in \mathcal{W}_{k}\right\}$.

Notice that each of $I, J, H$, and $E$ are contained in $\left(X_{j=1}^{k} \mathcal{W}_{k}\right) \times \mathcal{W}_{k+1}$, and consequently their closures in $Y$ and in $Z$ are identical.

2.4. Lemma. $\bar{E}$ is a subsemigroup of $Y, \bar{I}$ is an ideal of $\bar{E}, \bar{J}$ is a right ideal of $\bar{E}$, and $\bar{H}$ is a left ideal of $\bar{E}$.

Proof. [8 Theorems 2.22 and 4.17].

The proof of the following lemma uses an idea from [1]. This lemma is needed so that in Theorem 2.9 the cell of the partition $\mathcal{F}$ can be guaranteed to be central in $\mathcal{W}_{k+1}$. This fact is significant because, as we have noted, central sets are guaranteed to contain substantial combinatorial structures.

2.5. Lemma. $\bar{E} \cap K(Y) \neq \emptyset$ and so

$$
K(\bar{E})=\bar{E} \cap\left(\left(\searrow_{j=1}^{k} K\left(\beta \mathcal{W}_{k}\right)\right) \times K\left(\beta \mathcal{W}_{k+1}\right)\right)=\bar{E} \cap K(Y) .
$$

Proof. Let $s$ be a minimal idempotent in $\beta \mathcal{W}_{k}$ and pick by 8 , Theorem 1.60 and Corollary 2.6] an idempotent $p$ in $\mathcal{W}_{k+1}$ such that $p \leq s$. Let $\vec{p}=(s, s, \ldots, s, p)$.

We claim that it suffices to show that $\vec{p} \in \bar{I}$. Indeed, assume that we have done so. We have that $\vec{p} \in\left(X_{j=1}^{k} K\left(\beta \mathcal{W}_{k}\right)\right) \times K\left(\beta \mathcal{W}_{k+1}\right)$ and by $\underline{8}$, Theorem 2.23] $K(Y)=\left(\chi_{j=1}^{k} K\left(\beta \mathcal{W}_{k}\right)\right) \times K\left(\beta \mathcal{W}_{k+1}\right)$. Thus we have that $\vec{p} \in \bar{I} \cap K(Y) \subseteq$ $\bar{E} \cap K(Y)$. It then follows from [8, Theorem 1.65] that $K(\bar{E})=\bar{E} \cap K(Y)$.

To see that $\vec{p} \in \bar{I}$, let $A \in s$ and $B \in p$ be given. We need to show that $\left(\bar{A}^{k} \times \bar{B}\right) \cap I \neq \emptyset$. For each $t \in\{1,2, \ldots, k\}$ define $g_{t}: \mathcal{W}_{k+1} \rightarrow \mathcal{W}_{k}$ by letting $g_{t}(w)$ be the word obtained by replacing each occurrence of $k+1$ by an occurrence of $t$ and let $\widetilde{g}_{t}: \beta \mathcal{W}_{k+1} \rightarrow \beta \mathcal{W}_{k}$ be its continuous extension. Let $t \in\{1,2, \ldots, k\}$ be given. By [8, Corollary 4.22], $\widetilde{g}_{t}$ is a homomorphism and so $\widetilde{g_{t}}(p) \leq \widetilde{g_{t}}(s)$. Since $g_{t}$ is the identity on $\mathcal{W}_{k}$, we have that $\widetilde{g}_{t}(s)=s$, and thus $\widetilde{g}_{t}(p) \leq s$. Since $s$ is minimal, we therefore have that $\widetilde{g}_{t}(p)=s$.

Since $\bar{A}$ is a neighborhood of $\widetilde{g}_{t}(p)$ for each $t \in\{1,2, \ldots, k\}$, we have that $\bigcap_{t=1}^{k} g_{t}^{-1}[A] \in p$. Also, since $\beta \mathcal{W}_{k+1} \backslash \beta \mathcal{W}_{k}$ is an ideal of $\beta \mathcal{W}_{k+1}$ and $p$ is minimal in $\beta \mathcal{W}_{k+1}$, we have that $\mathcal{W}_{k+1} \backslash \mathcal{W}_{k} \in p$. Pick

$$
u \in\left(\mathcal{W}_{k+1} \backslash \mathcal{W}_{k}\right) \cap B \cap \bigcap_{t=1}^{k} g_{t}^{-1}[A]
$$

and let $w$ be the variable word over $\mathcal{W}_{k}$ which results from replacing each occurrence of $k+1$ by $v$. Then $w(k+1)=u$ and for each $t \in\{1,2, \ldots, k\}$ we have that $g_{t}(u)=$ $w(t)$. Consequently $(w(1), w(2), \ldots, w(k+1)) \in I \cap\left(A^{k} \times B\right)$ as required.

The proof of the following lemma uses an old idea of H. Furstenberg and Y. Katznelson in [5]. There is some redundancy in this lemma. For example, (5) and (13) both tell us that $p \cdot q_{k+1}=p$. 
2.6. Lemma. Let $s$ be any minimal idempotent in $\beta \mathcal{W}_{k}$. There exist minimal idempotents $p, q_{k+1}, r_{k+1} \in \beta \mathcal{W}_{k+1}$ and $q_{1}, q_{2}, \ldots, q_{k}, r_{1}, r_{2}, \ldots, r_{k} \in \beta \mathcal{W}_{k}$ such that:

(1) $\vec{p}=(s, s, \ldots, s, p) \in \bar{I}$;

(2) $\vec{q}=\left(q_{1}, q_{2}, \ldots, q_{k+1}\right) \in \bar{J}$

(3) $\vec{r}=\left(r_{1}, r_{2}, \ldots, r_{k+1}\right) \in \bar{H}$;

(4) $p=p \cdot s=s \cdot p$;

(5) $\vec{p} \cdot \vec{q}=\vec{p}$ and $\vec{q} \cdot \vec{p}=\vec{q}$;

(6) $\vec{p} \cdot \vec{r}=\vec{r}$ and $\vec{r} \cdot \vec{p}=\vec{p}$;

(7) for $i \in\{1,2, \ldots, k+1\}$ and $j \in\{1,2, \ldots, k\}, q_{i} \cdot q_{j}=q_{i}$;

(8) for $i \in\{1,2, \ldots, k+1\}$ and $j \in\{1,2, \ldots, k\}, r_{j} \cdot r_{i}=r_{i}$;

(9) $s \cdot q_{k+1}=p$;

(10) $r_{k+1} \cdot s=p$;

(11) $q_{k+1} \cdot s=q_{k+1}$;

(12) $s \cdot r_{k+1}=r_{k+1}$;

(13) for $j \in\{1,2, \ldots, k+1\}, p \cdot q_{j}=p$;

(14) for $j \in\{1,2, \ldots, k+1\}, r_{j} \cdot p=p$;

(15) for $j \in\{1,2, \ldots, k\}, q_{j} \cdot q_{k+1}=q_{j} \cdot p$; and

(16) for $j \in\{1,2, \ldots, k\}, r_{k+1} \cdot r_{j}=p \cdot r_{j}$.

Proof. Let $\bar{s}=(s, s, \ldots, s)$. Then $\bar{s} \in \bar{E}$. By [8] Corollary 2.6 and Theorem 2.7], every left ideal of $\bar{E}$ contains a minimal left ideal and every right ideal of $\bar{E}$ contains a minimal right ideal. Pick a minimal left ideal $L$ of $\bar{E}$ with $L \subseteq \bar{H}$ and a minimal right ideal $R$ of $\bar{E}$ with $R \subseteq \bar{J}$. Pick by Lemma 2.1 a minimal idempotent $\vec{p} \leq \bar{s}$ in $\bar{E}$ with $\vec{p} \in L \bar{s} \cap \bar{s} R$.

Now $\vec{p}=\left(p_{1}, p_{2}, \ldots, p_{k+1}\right)$. Since $\vec{p} \leq \bar{s}$, we have that for each $t \in\{1,2, \ldots$, $k+1\}, p_{t} \leq s$. Since $s$ is minimal in $\beta \mathcal{W}_{k}$, we have in particular that $p_{t}=s$ for $t \in\{1,2, \ldots, k\}$. Let $p=p_{k+1}$. Then we have $\vec{p}=(s, s, \ldots, s, p)$. Since $\vec{p} \in K(\bar{E})$ and, by Lemma 2.5 $K(\bar{E})=\bar{E} \cap\left(X_{j=1}^{k} K\left(\beta \mathcal{W}_{k}\right) \times K\left(\beta \mathcal{W}_{k+1}\right)\right)$, we have that $p$ is minimal in $\beta \mathcal{W}_{k+1}$. Since $p \leq s$, we have that (4) holds. Since $\vec{p}$ is minimal in $\bar{E}$ and $\bar{I}$ is an ideal of $\bar{E}$, we have that $\vec{p} \in \bar{I}$. That is, (1) holds.

Since $\vec{p} \in L \bar{s} \cap \bar{s} R$, pick $\vec{m} \in L$ and $\vec{n} \in R$ such that $\vec{p}=\vec{m} \bar{s}=\bar{s} \vec{n}$. Then $p=m_{k+1} s=s n_{k+1}$ and for $j \in\{1,2, \ldots, k\}, s=m_{j} s=s n_{j}$. Also $m_{k+1} p=$ $m_{k+1} s p=p p=p$ and $p n_{k+1}=p$. For $j \in\{1,2, \ldots, k\}$, we have that $m_{j} p=$ $m_{j} s p=s p=p$ and $p n_{j}=p$. Let $\vec{q}=\vec{n} \vec{p}$ and let $\vec{r}=\vec{p} \vec{m}$. Now $q_{k+1} q_{k+1}=$ $n_{k+1} p n_{k+1} p=n_{k+1} p p=n_{k+1} p=q_{k+1}$. The fact that $q_{j}$ is an idempotent for each $j \in\{1,2, \ldots, k\}$ follows from (7) which we shall verify below. Likewise, for each $j \in\{1,2, \ldots, k+1\}, r_{j} r_{j}=r_{j}$.

Since $\vec{n} \in R$ and $\vec{m} \in L$ we have $\vec{q} \in R \subseteq \bar{J}$ and $\vec{r} \in R \subseteq \bar{H}$. Thus (2) and (3) hold. Also $\vec{q} \in R \subseteq K(\bar{E}) \subseteq \chi_{j=1}^{k} K\left(\beta \mathcal{W}_{k}\right) \times K\left(\beta \mathcal{W}_{k+1}\right)$, so we have that $q_{k+1}$ is minimal in $\beta \mathcal{W}_{k+1}$ and $q_{j}$ is minimal in $\beta \mathcal{W}_{k}$ for each $j \in\{1,2, \ldots, k\}$. Similarly, $r_{k+1}$ is minimal in $\beta \mathcal{W}_{k+1}$ and $r_{j}$ is minimal in $\beta \mathcal{W}_{k}$ for each $j \in\{1,2, \ldots, k\}$.

We proceed to verify the odd numbered statements from (5) through (15), the corresponding even numbered statements being analogous.

Since $\vec{p}=\bar{s} \vec{n}$ and $p n_{k+1}=p$, we have that $\vec{p}=\vec{p} \vec{n}$. Therefore we have $\vec{p} \vec{q}=$ $\vec{p} \vec{n} \vec{p}=\vec{p} \vec{p}=\vec{p}$ and $\vec{q} \vec{p}=\vec{n} \vec{p} \vec{p}=\vec{n} \vec{p}=\vec{q}$. Thus (5) holds.

To verify statement (7), first let $i, j \in\{1,2, \ldots, k\}$. Then $q_{i} q_{j}=n_{i} s q_{j}=n_{i} s=$ $q_{i}$. Now let $j \in\{1,2, \ldots, k\}$. Then $q_{k+1} q_{j}=n_{k+1} p n_{j} s=n_{k+1} p s=n_{k+1} p=q_{k+1}$. 
Since $s q_{k+1}=s n_{k+1} p=p p=p$ we have that (9) holds. For statement (11), we have $q_{k+1} s=n_{k+1} p s=n_{k+1} p=q_{k+1}$. To verify (13), let $j \in\{1,2, \ldots, k\}$. Then $p q_{j}=p n_{j} s=p s=p$. From (5) we know that $p q_{k+1}=p$. To verify (15), let $j \in\{1,2, \ldots, k\}$. Then $q_{j} q_{k+1}=n_{j} s n_{k+1} p=n_{j} p p=n_{j} p=n_{j} s p=q_{j} p$.

In Theorem 2.9 we shall be choosing a sequence of variable words $\left\langle w_{n}\right\rangle_{n=1}^{\infty}$ such that whenever $n \equiv 1(\bmod 3), w_{n}$ is a right variable word and whenever $n \equiv$ $0(\bmod 3), w_{n}$ is a left variable word. We shall expect certain products of these words to lie in specified cells of finite partitions of $\mathcal{W}_{k}$ and of $\mathcal{W}_{k+1} \backslash \mathcal{W}_{k}$. We clearly cannot have the first word of such a product be a left variable word nor can we have the last word be a right variable word, since one may divide $\mathcal{W}_{k}$ according to the first or last letter. Nor can we allow a right variable word to be followed by a left variable word, since that allows manipulation of adjacent occurrences of letters. (See [7] for a fuller discussion of these points.)

2.7. Definition. A set $F \in \mathcal{P}_{f}(\mathbb{N})$ is allowable if and only if $\max F \not \equiv 1(\bmod 3)$, $\min F \not \equiv 0(\bmod 3)$, and for all $i<j$ in $F$, if $i \equiv 1(\bmod 3)$ and $j \equiv 0(\bmod 3)$, then there exists $h \in F$ such that $i<h<j$ and $h \equiv 2(\bmod 3)$.

Notice that if in the following lemma $F$ is allowable, then conclusion (1) or (2) applies.

2.8. Lemma. Let $F \in \mathcal{P}_{f}(\mathbb{N})$ and assume that $\max F \not \equiv 1(\bmod 3)$ and for all $i<j$ in $F$, if $i \equiv 1(\bmod 3)$ and $j \equiv 0(\bmod 3)$, then there exists $h \in F$ such that $i<h<j$ and $h \equiv 2(\bmod 3)$. Let $s, p, \vec{p}=\left(p_{1}, p_{2}, \ldots, p_{k+1}\right), \vec{q}=$ $\left(q_{1}, q_{2}, \ldots, q_{k+1}\right)$, and $\vec{r}=\left(r_{1}, r_{2}, \ldots, r_{k+1}\right)$ be as in Lemma 2.6 (so that $p_{k+1}=p$ and $p_{j}=s$ for $\left.j \in\{1,2, \ldots, k\}\right)$. For $f: F \rightarrow\{1,2, \ldots, k+1\}$ and $n \in F$, define

$$
\phi(f, n)= \begin{cases}q_{f(n)} & \text { if } n \equiv 0(\bmod 3), \\ r_{f(n)} & \text { if } n \equiv 1(\bmod 3), \\ p_{f(n)} & \text { if } n \equiv 2(\bmod 3) .\end{cases}
$$

(1) If $\min F \not \equiv 0(\bmod 3)$ and $k+1 \notin \operatorname{range}(f)$, then $\prod_{n \in F} \phi(f, n)=s$.

(2) If $\min F \not \equiv 0(\bmod 3)$ and $k+1 \in \operatorname{range}(f)$, then $\prod_{n \in F} \phi(f, n)=p$.

(3) If $\min F \equiv 0(\bmod 3)$ and $k+1 \notin \operatorname{range}(f)$, then $\prod_{n \in F} \phi(f, n) \in\left\{q_{1}, q_{2}, \ldots\right.$, $\left.q_{k}\right\}$.

(4) If $\min F \equiv 0(\bmod 3)$ and $k+1 \in \operatorname{range}(f)$, then $\prod_{n \in F} \phi(f, n) \in\left\{q_{k+1}\right\} \cup$ $\left\{q_{1} p, q_{2} p, \ldots, q_{k} p\right\}$.

Proof. We proceed by induction on $|F|$. If $F=\{m\}$, then $m \equiv 0(\bmod 3)$ or $m \equiv 2(\bmod 3)$. If $m \equiv 0(\bmod 3)$, then $\phi(f, m)$ is $q_{f(m)}$ and if $m \equiv 2(\bmod 3)$, then $\phi(f, m)$ is $p_{f(m)}$.

Now assume that $|F|>1$, let $m=\min F$, and let $G=F \backslash\{m\}$. Let $l=\min G$. Then the value of $\prod_{n \in G} \phi(f, n)$ is determined by $f_{\mid G}$ and the congruence class of $l$ using the induction hypothesis. The conclusions then follow from Lemma 2.6 and the fact that one cannot have both $m \equiv 1(\bmod 3)$ and $l \equiv 0(\bmod 3)$.

Notice that in the following theorem, which is our main result, the fact that $A$ can be any central subset of $\mathcal{W}_{k}$ yields a stronger result than choosing some $A$ out of a given finite partition of $\mathcal{W}_{k}$, because for any finite partition, some cell must be central. Notice also that one cannot reverse the roles of $A$ and $B$ by taking $B$ to be an arbitrary central set in $\mathcal{W}_{k+1}$ and picking $A$ out of a finite partition of 
$\mathcal{W}_{k}$. Indeed, let $R=(k+1) \mathcal{W}_{k+1}$. Then $R$ is a right ideal of $\mathcal{W}_{k+1}$ and so by 8, Corollary 4.18] $\bar{R}$ is a right ideal of $\beta \mathcal{W}_{k+1}$, so that $R$ is central. Given the sequence $\left\langle w_{n}\right\rangle_{n=1}^{\infty}$ of variable words over $\mathcal{W}_{k}$ as chosen in Theorem 2.9 one cannot have $w_{1}(1) w_{2}(k+1) \in R$.

2.9. Theorem. Let $A$ be a central subset of $\mathcal{W}_{k}$ and let $\mathcal{F}$ be a finite partition of $\mathcal{W}_{k+1} \backslash \mathcal{W}_{k}$. Then there exist $B \in \mathcal{F}$ such that $B$ is central in $\mathcal{W}_{k+1}$ and a sequence $\left\langle w_{n}\right\rangle_{n=1}^{\infty}$ of variable words over $\mathcal{W}_{k}$ such that:

(1) for each $n \in \mathbb{N}$, if $n \equiv 1(\bmod 3)$, then $w_{n}$ is a right variable word;

(2) for each $n \in \mathbb{N}$, if $n \equiv 0(\bmod 3)$, then $w_{n}$ is a left variable word; and

(3) for every allowable $F \in \mathcal{P}_{f}(\mathbb{N})$ and every $h: F \rightarrow\{1,2, \ldots, k+1\}$, if $k+1 \in$ $h[F]$, then $\prod_{n \in F} w_{n}(h(n)) \in B$, and if $k+1 \notin h[F]$, then $\prod_{n \in F} w_{n}(h(n)) \in$ $A$.

Proof. Pick a minimal idempotent $s \in \beta \mathcal{W}_{k}$ such that $A \in p$. Pick $\vec{p}=\left(p_{1}, p_{2}, \ldots\right.$, $\left.p_{k+1}\right), \vec{q}=\left(q_{1}, q_{2}, \ldots, q_{k+1}\right)$, and $\vec{r}=\left(r_{1}, r_{2}, \ldots, r_{k+1}\right)$ as guaranteed by Lemma 2.6. Notice that, with $p$ as in Lemma[2.6] we have $p_{k+1}=p$ and for $j \in\{1,2, \ldots, k\}$, $p_{j}=s$. Since $p$ is minimal in $\mathcal{W}_{k+1}$ and $\mathcal{W}_{k+1} \backslash \mathcal{W}_{k}$ is an ideal of $\mathcal{W}_{k+1}$, we have that $\mathcal{W}_{k+1} \backslash \mathcal{W}_{k} \in p$. Pick $B \in \mathcal{F}$ such that $B \in p_{k+1}$. Let $C=A \cup B$ and notice that $\vec{p} \in c \ell_{Z}\left(X_{j=1}^{k+1} C\right)$.

Let $L=\left\{e, p, s, q_{1}, q_{2}, \ldots, q_{k+1}, q_{1} p, q_{2} p, \ldots, q_{k+1} p\right\}$. For notational convenience, let $p_{0}=q_{0}=r_{0}=e$. Also, if $w$ is a variable word, we let $w(0)=e$. If $n \in \mathbb{N}$, $f:\{1,2, \ldots, 3 n\} \rightarrow\{0,1, \ldots, k+1\}$, and $i \in\{1,2, \ldots, 3 n\}$, define $\phi(f, i)$ as in Lemma 2.8 (with the obvious extension of the definition when $f(i)=0$ ). Given $\theta \in L$, we say that the pair $(f, \theta)$ is admissible if and only if for every $j \in\{1,2, \ldots$, $3 n\}$, we have $\left(\prod_{i=j}^{3 n} \phi(f, j)\right) \theta \in L$. Notice that, if $n>1,(f, \theta)$ is admissible, and $g$ is the restriction of $f$ to $\{1,2, \ldots, 3 n-3\}$, then $\left(g, r_{f(3 n-2)} p_{f(3 n-1)} q_{f(3 n)} \theta\right)$ is also admissible.

We construct the sequence $\left\langle w_{n}\right\rangle_{n=1}^{\infty}$ inductively, three terms at a time. Let $W_{0}=$ $\{e\}$ and for each $i \in \mathbb{N}$, as soon as we have chosen $w_{i}$, let $W_{i}=\left\{\prod_{j=1}^{i} w_{j}(f(j))\right.$ : $f:\{1,2, \ldots, i\} \rightarrow\{0,1, \ldots, k+1\}\}$.

Let $n \in \mathbb{N} \cup\{0\}$ and assume that we have chosen $w_{i}$ for all $i \in \mathbb{N}$ with $i \leq 3 n$ (if any) so that, if $n \geq 1, \theta \in L, f:\{1,2, \ldots, 3 n\} \rightarrow\{0,1, \ldots, k+1\}$, and $(f, \theta)$ is admissible, then

$$
\left(\prod_{i=1}^{3 n} w_{i}(f(i))\right)^{-1} C \in \theta \Leftrightarrow C \in\left(\prod_{i=0}^{n-1} r_{f(3 i+1)} p_{f(3 i+2)} q_{f(3 i+3)}\right) \theta .
$$

Now $\vec{r} \in \bar{H}$, so pick by Lemma 2.2 a right variable word $w_{3 n+1}$ over $\mathcal{W}_{k}$ such that for all $l \in L$, all $u \in W_{3 n}$, and all $t \in\{1,2, \ldots, k+1\}$,

$$
\left(u w_{3 n+1}(t)\right)^{-1} C \in l \Leftrightarrow u^{-1} C \in r_{t} l .
$$

Since $w_{3 n+1}(0)=e=r_{0}$ we have also that (a) holds for $t=0$.

Since $\vec{p} \in \bar{I}$, pick by Lemma 2.2 (and the observation above about the case $t=0)$ a variable word $w_{3 n+2}$ over $\mathcal{W}_{k}$ such that for all $l \in L$, all $u \in W_{3 n+1}$, and all $t \in\{0,1, \ldots, k+1\}$,

$$
\left(u w_{3 n+2}(t)\right)^{-1} C \in l \Leftrightarrow u^{-1} C \in p_{t} l .
$$


Since $\vec{q} \in \bar{J}$, pick by Lemma $\left[2.2\right.$ a left variable word $w_{3 n+3}$ over $\mathcal{W}_{k}$ such that for all $l \in L$, all $u \in W_{3 n+2}$, and all $t \in\{0,1, \ldots, k+1\}$,

$$
\left(u w_{3 n+3}(t)\right)^{-1} C \in l \Leftrightarrow u^{-1} C \in q_{t} l .
$$

Now let $\theta \in L$, let $f:\{1,2, \ldots, 3 n+3\} \rightarrow\{0,1, \ldots, k+1\}$, and assume that $(f, \theta)$ is admissible. We verify that $(*)$ holds. Notice that, since $(f, \theta)$ is admissible, we have that

$$
\left\{q_{f(3 n+3)} \theta, p_{f(3 n+2)} q_{f(3 n+3)} \theta, r_{f(3 n+1)} p_{f(3 n+2)} q_{f(3 n+3)} \theta\right\} \subseteq L .
$$

(In the following, if $n=0$ we interpret $\prod_{i=1}^{3 n} w_{i}(f(i))$ as e.)

$$
\begin{aligned}
& \left(\prod_{i=1}^{3 n+3} w_{i}(f(i))\right)^{-1} C \in \theta \\
\Leftrightarrow & \left(\left(\prod_{i=1}^{3 n} w_{i}(f(i))\right) w_{3 n+1}(f(3 n+1)) w_{3 n+2}(f(3 n+2))\right)^{-1} C \in q_{f(3 n+3)} \theta \\
\Leftrightarrow & \left(\left(\prod_{i=1}^{3 n} w_{i}(f(i))\right) w_{3 n+1}(f(3 n+1))\right)^{-1} C \in p_{f(3 n+2)} q_{f(3 n+3)} \theta \\
\Leftrightarrow & \left(\prod_{i=1}^{3 n} w_{i}(f(i))\right)^{-1} C \in r_{f(3 n+1)} p_{f(3 n+2)} q_{f(3 n+3)} \theta \\
\Leftrightarrow & C \in\left(\prod_{i=0}^{n} r_{f(3 i+1)} p_{f(3 i+2)} q_{f(3 i+3)}\right) \theta .
\end{aligned}
$$

Here the first three double implications hold by (c), (b), and (a) respectively. If $n=0$, the last double implication is a tautology. Otherwise, it is a consequence of the induction hypothesis.

The construction being complete, we claim that the sequence $\left\langle w_{n}\right\rangle_{n=1}^{\infty}$ is as required. The first two conclusions are immediate. So let $F \in \mathcal{P}_{f}(\mathbb{N})$ be allowable and let $h: F \rightarrow\{1,2, \ldots, k+1\}$. Choose the least $n \in \mathbb{N}$ such that $F \subseteq\{1,2, \ldots$, $3 n\}$. Define $f:\{1,2, \ldots, 3 n\} \rightarrow\{0,1, \ldots, k+1\}$ by

$$
f(i)= \begin{cases}h(i) & \text { if } i \in F \\ 0 & \text { if } i \in\{1,2, \ldots, 3 n\} \backslash F .\end{cases}
$$

Then we have immediately that $\prod_{i \in F} w_{i}(h(i))=\prod_{i=1}^{3 n} w_{i}(f(i))$.

By Lemma 2.8 we have that the pair $(f, e)$ is admissible. Recall that we identify $e$ with the principal ultrafilter generated by $e$. Thus by $(*)$, we have that

$$
\prod_{i=1}^{3 n} w_{i}(f(i)) \in C \Leftrightarrow C \in \prod_{i=0}^{n-1} r_{f(3 i+1)} p_{f(3 i+2)} q_{f(3 i+3)} .
$$

Again by Lemma 2.8 we have that

$$
\prod_{i=0}^{n-1} r_{f(3 i+1)} p_{f(3 i+2)} q_{f(3 i+3)}= \begin{cases}s & \text { if } k+1 \notin \operatorname{range}(f), \\ p & \text { if } k+1 \in \operatorname{range}(f) .\end{cases}
$$

In either case, we have that $C \in \prod_{i=0}^{n-1} r_{f(3 i+1)} p_{f(3 i+2)} q_{f(3 i+3)}$, and so $\prod_{i=1}^{3 n} w_{i}(f(i))$ $\in C$. If $k+1 \notin \operatorname{range}(h)$, then $\prod_{i=1}^{3 n} w_{i}(f(i)) \in C \cap \mathcal{W}_{k}=A$. If $k+1 \in \operatorname{range}(h)$, then $\prod_{i=1}^{3 n} w_{i}(f(i)) \in C \backslash \mathcal{W}_{k}=B$. 
We conclude by showing that Carlson's Main Lemma is a consequence of Theorem [2.9.

2.10. Corollary (Carlson). Let $k \in \mathbb{N}$, let the set of variable words over $\mathcal{W}_{k}$ be partitioned into finitely many classes, and let $\left\langle w_{n}\right\rangle_{n=1}^{\infty}$ be a sequence of variable words. Then there exists a variable reduction $\left\langle t_{n}\right\rangle_{n=1}^{\infty}$ of $\left\langle w_{n}\right\rangle_{n=1}^{\infty}$ such that all expressions of the form $\prod_{i=1}^{n} t_{i}(f(i))$, where $n \in \mathbb{N}, f:\{1,2, \ldots, n\} \rightarrow\{1,2, \ldots$, $k\} \cup\{v\}$, and $v \in \operatorname{range}(f)$, lie in the same cell of the partition.

Proof. Let $\mathcal{W}_{k, v}$ be the set of variable words over $\mathcal{W}_{k}$, let $\mathcal{F}$ be a finite partition of $\mathcal{W}_{k, v}$, and let $\left\langle w_{n}\right\rangle_{n=1}^{\infty}$ be a sequence of variable words. Define a function $\varphi$ : $\mathcal{W}_{k+1} \backslash \mathcal{W}_{k} \rightarrow \mathcal{W}_{k, v}$ as follows. If $u=a_{1} a_{2} \cdots a_{l}$, where each $a_{i} \in\{1,2, \ldots, k+1\}$, let $b_{i}=a_{i}$ if $a_{i} \neq k+1$ and $b_{i}=v$ if $a_{i}=k+1$; then let $\varphi(u)=\prod_{i=1}^{l} w\left(b_{i}\right)$.

Let $\mathcal{F}=\left\{\varphi^{-1}[H]: H \in \mathcal{F}\right\}$. Then $\mathcal{F}$ is a finite partition of $\mathcal{W}_{k+1} \backslash \mathcal{W}_{k}$. Let $A$ be any central subset of $\mathcal{W}_{k}$ and pick $B \in \mathcal{F}$ and a sequence $\left\langle w_{n}^{\prime}\right\rangle_{n=1}^{\infty}$ of variable words as guaranteed by Theorem [2.9. Pick $H \in \mathcal{F}$ such that $B=\varphi^{-1}[H]$.

For each $n \in \mathbb{N}$, let $l_{n}$ be the length of $w_{3 n-1}^{\prime}$ and write $w_{3 n-1}^{\prime}=a_{n, 1} a_{n, 2} \cdots a_{n, l_{n}}$ where each $a_{n, i} \in\{1,2, \ldots, k\} \cup\{v\}$. (For this corollary, we are avoiding both left and right variable words.) Let $\alpha_{0}=0$ and for $n \in \mathbb{N}$, let $\alpha_{n}=\sum_{m=1}^{n} l_{m}$. For each $n \in \mathbb{N}$, let $t_{n}=\prod_{m=1}^{l_{n}} w_{\alpha_{n-1}+m}\left(a_{n, m}\right)$. Then $\left\langle t_{n}\right\rangle_{n=1}^{\infty}$ is a variable reduction of $\left\langle w_{n}\right\rangle_{n=1}^{\infty}$.

To see that $\left\langle t_{n}\right\rangle_{n=1}^{\infty}$ is as required, let $n \in \mathbb{N}$ and let $f:\{1,2, \ldots, n\} \rightarrow\{1,2$, $\ldots, k\} \cup\{v\}$ with $v \in \operatorname{range}(f)$. Define $g:\{1,2, \ldots, n\} \rightarrow\{1,2, \ldots, k+1\}$ by $g(i)=f(i)$, if $f(i) \neq v$ and $g(i)=k+1$ if $f(i)=v$. Then $\prod_{i=1}^{n} w_{3 i-1}^{\prime}(g(i)) \in B$ and so $\prod_{i=1}^{n} t_{i}(f(i))=\varphi\left(\prod_{i=1}^{n} w_{3 i-1}^{\prime}(g(i))\right) \in H$.

\section{REFERENCES}

[1] V. Bergelson, A. Blass, and N. Hindman, Partition theorems for spaces of variable words, Proc. London Math. Soc. 68 (1994), 449-476. MR 95i:05107

[2] T. Carlson, Some unifying principles in Ramsey Theory, Discrete Math. 68 (1988), 117-169. MR 89b:04006

[3] T. Carlson and S. Simpson, A dual form of Ramsey's Theorem, Advances in Math. 53 (1984), 265-290. MR 85h:04002

[4] H. Furstenberg, Recurrence in ergodic theory and combinatorial number theory, Princeton University Press, 1981. MR 82j:28010

[5] H. Furstenberg and Y. Katznelson, Idempotents in compact semigroups and Ramsey Theory, Israel J. Math. 68 (1989), 257-270. MR 92d:05170

[6] A. Hales and R. Jewett, Regularity and positional games, Trans. Amer. Math. Soc. 106 (1963), 222-229. MR 26:1265

[7] N. Hindman and R. McCutcheon, Partition theorems for left and right variable words, manuscript.

[8] N. Hindman and D. Strauss, Algebra in the Stone-Čech compactification, Walter de Gruyter, Berlin, 1998. MR 99j:54001

[9] R. McCutcheon, Two new extensions of the Hales-Jewett Theorem, Electronic J. of Combinatorics 7 (2000), R49. MR 2001i:05151

Department of Mathematics, Howard University, Washington, DC 20059

E-mail address: nhindman@fac.howard.edu

E-mail address: nhindman@aol.com

URL: http://members.aol.com/nhindman/

Department of Mathematical Sciences, University of Memphis, Memphis, Tennessee 38152

E-mail address: randall@msci.memphis.edu 